\title{
Computer aided drug design of florfenicol to target chloramphenicol acetyltransferase of vibriosis causing pathogens
}

\author{
Ratul Bhowmik ${ }^{1}$, Shubham Roy ${ }^{1}$, Sounok Sengupta ${ }^{2}$, Lokesh Ravi ${ }^{3 *}$ (i) \\ ${ }^{1}$ Department of Pharmaceutical Chemistry, SPER, Jamia Hamdard, New Delhi, India. \\ ${ }^{2}$ Department of Pharmacology, NSHM Knowledge Campus, Kolkata-Group of Institutions, Kolkata, India. \\ ${ }^{3}$ Department of Botany, St. Josephs College (Autonomous), Bangalore, Karnataka, India.
}

\section{ARTICLE INFO \\ Article history: \\ Received on: June 25, 2021 \\ Accepted on: September 21, 2021 \\ Available online: January 07, 2022}

\section{Key words:}

Vibriosis, florfenicol, acetyltransferase, Structure

Based Computer Aided Drug

Design (SBCADD), Absorption,

Distribution, Metabolism,

Excretion and Toxicity (ADMET)

\begin{abstract}
Vibriosis, a bacterial infection, is very much responsible for causing significant losses in the aquaculture industry. Chloramphenicol acetyltransferase is an antibiotic resistant enzyme present in the vibrio class of bacteria. Florfenicol, a benzene sulphonyl antibiotic is the lead molecule in this study. OSIRIS property explorer, Chemsketch, Molinspiration, pre ADMET tools were used for ligand modification, while AutoDock Vina, PyMOL, and LigPlot+ were used for protein-ligand docking analysis. Florfenicol demonstrated a free binding energy of $-6.0 \mathrm{kcal} / \mathrm{mol}$ and poor ADMET properties. Ligand structures were optimized using "uff" forcefield and conjugate gradients algorithm. Primary drug designing was done with emphasis on ADMET properties that yielded a total of 15 modified ligands. Among these 15 ligands, F006 exhibited the highest dock score of -7.3 $\mathrm{kcal} / \mathrm{mol}$ along with significant ADME properties and was hence chosen for further secondary modifications. Ligand F006 was further modified on the basis of ADMET properties to obtain 17 modified ligands. Among the 17 secondary ligands, F016 demonstrated strong binding affinity of $-8.6 \mathrm{kcal} / \mathrm{mol}$ and also demonstrated significant ADME properties. Based on the results of this studies, the structure based computational drug design of florfenicol concluded that the modified ligand F016 designed in this study has good ADMET properties along with strong binding affinity towards the drug target chloramphenicol acetyltransferase protein and could be a potential solution in treating vibriosis.
\end{abstract}

\section{INTRODUCTION}

Vibriosis is a typical systemic bacterial type of infection. It is caused by bacteria belonging to the family Vibrionaceae. Vibriosis is often considered a significant problem worldwide in the aquaculture industry. The common factors behind the cause of vibriosis in fishes are fluctuation of physicochemical properties of water as well as overpopulation. Previously, vibriosis has been responsible for causing significant economic loss in the shrimp aquaculture industry. Recently, vibriosis has also been responsible for causing losses associated with various species of cage-cultured fishes worldwide $[1,2]$.

\footnotetext{
*Corresponding Author

Lokesh Ravi, Department of Botany, St. Josephs College (Autonomous), Bangalore, Karnataka, India.E-mail: lokesh.ravi@sjc.ac.in
}

The vibrio class of bacteria has been reported to interfere with the mode of action of various drugs, especially antibiotics. They act by modifying the functional group of the antibiotics, thereby rendering them ineffective $[3,4]$. Chloramphenicol acetyltransferase is one such antibiotic resistance enzyme present in the vibrio class of bacteria. This particular enzyme of the bacteria provides resistance against chloramphenicol type of drugs by acetylating the C-3 hydroxyl group of the drug, thereby preventing them from binding to the $50 \mathrm{~S}$ subunit of the bacterial ribosome. Thus, the bacterial protein translation process is not stopped $[5,6]$.

Florfenicol is one such drug that has been specifically used to treat vibriosis worldwide. Florfenicol antibiotic primarily provides bacteriostatic action and has been reported to show similar activity as that of chloramphenicol $[7,8]$. Previous researches have stated that florfenicol functions by binding to the bacterial ribosomal subunits leading to inhibition of the bacterial acetyltransferase enzyme. Moreover, the bacterial receptor target of action of 
florfenicol has been found to be the same as that of thiamphenicol and chloramphenicol. Furthermore, the structure of florfenicol contains fluorine atoms in the place of hydroxyl group at $\mathrm{C}-3$ position in the structure of thiamphenicol and chloramphenicol. The presence of the fluorine group helps florfenicol to be less susceptible to bacterial deactivation, thereby making it more effective than chloramphenicol and thiamphenicol. In spite of all this positivity, florfenicol has been found to show poor drug properties as well as adverse effects $[9,10]$.

The main aim to be achieved through this study is to design new analogs of florfenicol that can target bacterial acetyltransferase and exhibit better drug properties as well as fewer side effects. Using florfenicol as the base framework, the primary and secondary modifications were done by using Chemsketch software. All these modified molecules were then screened against all the drug-likeness rules. Following this, ADMET analysis of the modified molecules is also performed. After these steps, the configured molecules were docked in the active sites of the bacterial acetyltransferase enzyme. The results obtained from the study showed that the designed molecules can be considered as potential lead molecules in inhibiting bacterial chloramphenicol acetyltransferase enzymes, which could ultimately be used to treat vibriosis [11,12].

\section{MATERIALS AND METHODS}

\subsection{Molecular Target and Active Site Determination}

The bacterial chloramphenicol acetyltransferase is the main target which is responsible for providing resistance against antibiotics administered in vibriosis. The molecular target structure was retrieved from the Protein Data Bank (PDB). The name of the PDB-ID retrieved for the study is 6PXA. The protein 6PXA contains preexisting native ligand chloramphenicol that shows the active pockets. The unnecessary native ligands and water molecules were first cleaned using PyMOL software $[13,14]$. The binding pockets present on the protein were visualized using PyMOL \& Discovery Studio, based on the co-crystallized ligands and also the inbuilt AutoDock Vina protocol that predicts the binding site during protein-ligand docking process $[15,16]$.

\subsection{Docking of Florfenicol with Chloramphenicol Acetyltransferase}

The Florfenicol molecule was used as the basic framework for designing effective lead molecules. The structure of Florfenicol was retrieved from the pubchem database in the form of SDF format. Ligand structure was optimized using "uff" forcefield and conjugate gradients algorithm, available within PyRx-Open Babel tool. This structure was then docked with the molecular target chloramphenicol acetyltransferase. The binding affinity obtained from docking is then used as the standard reference for further modified structures. The docking study was done using PyRx (Autodock Vina), computational drug discovery and virtual screening software used for screening libraries of compounds against potential drug targets [14,17].

\subsection{Initial Ligand Modifications Using Florfenicol Framework}

Different molecules were designed using florfenicol as the basic framework for modification. The molecules were drawn and designed using Chemsketch software. The newly sketched molecules were then subjected to various in silico analysis. Basically, to fit perfectly into the active pocket of the protein, initial lead modifications were performed.

\subsection{Primary Lead Development and Drug Screening}

The OSIRIS property explorer tool was used to modify the lead molecule. The structure of florfenicol was assessed in the stand-alone Java Runtime Environment. It was then modified by substitution or deletion or by the addition of atoms in a manner such that the skeleton structure of the lead molecule was retained. The modifications were also done to increase both solubility and polarity as well as reducing unnecessary side effects. Using Lipinski's rule of five, ADMET analysis was done using physiological parameters such as LogP, solubility, topological polar surface area (TPSA), molecular weight, drug likeness, and drug score. All the values were noted. The color code indicated the severity of the unnecessary side effect. The green color indicated no unnecessary side effects. The red color indicated severe side effects. Orange and yellow colors denoted medium and low risk of unnecessary side effects respectively. The better properties showing designed molecules were then draw in Chemsketch. Following this, the molecules were saved in a PDB format for further docking analysis.

\subsection{Docking and Selection of the Lead Molecule}

The docking procedure was done using PyRx (Autodock Vina). The proteins were prepared first using Autodock tools. After this step, the protein was introduced into PyRx. The modified molecules were then introduced into PyRx. The energy minimization of the molecules was done using "uff" forcefield and conjugate gradients algorithm, available within PyRx-Open Babel tool. Following this, docking was performed. The results were displayed in terms of binding affinity. The binding affinity represents the binding energy. The binding energy exhibits the extent of binding of a particular molecule. Furthermore, the best type of configuration would be the one that would bind with its target. The binding affinity of chloramphenicol acetyltransferase with the initially modified ligands should be more than the binding affinity of florfenicol with chloramphenicol acetyltransferase. The molecule with the best binding affinity and good ADME property was chosen for further analysis.

\subsection{Secondary Ligand Modifications of Initial Ligand}

Further alterations were made to design new lead molecules using the best initial ligand modification. The secondary lead modifications were aimed for increasing stability. This was mainly done for acquiring better binding affinity between new lead molecules with chloramphenicol acetyltransferase. The structures were drawn, modified, and designed using Chemsketch. Following this, the molecules were subjected to in silico analysis similarly as that of the initial ligand modifications.

\subsection{Secondary Lead Development and Drug Screening}

The secondary ligand modifications were also designed using OSIRIS property explorer tool. The designed molecules were then assessed in the stand-alone Java Runtime Environment. The molecules were 
designed in such a manner so that the skeletal structure of the lead molecule was retained. Furthermore, modifications were aimed at improving polarity and solubility as well as reducing unnecessary side effects. Using Lipinski's rule of five, the ADME analysis of the secondary lead molecules was performed. Physicochemical parameters such as LogP, solubility, TPSA, molecular weight drug-likeness, and drug score were all noted down. The color code indicated the severity of the unnecessary side effect. The green color indicated no unnecessary side effects. The red color indicated severe side effects. Orange and yellow colors denoted medium and low risk of unnecessary side effects, respectively. The better properties showing designed molecules were then drawn in Chemsketch. Following this, the molecules were saved in the PDB format for further docking analysis.

\subsection{Docking and Selection of Lead Molecule}

The docking study was carried out using PyRx (Autodock Vina). The secondary ligand modifications were docked with the chloramphenicol acetyltransferase receptor. The binding affinity obtained from docking should be higher than that binding affinity of the initial lead modifications and the chloramphenicol acetyltransferase receptor. The lead molecules were selected based on higher binding affinity and better ADME properties than primary ligand modifications. More drug properties of the best lead molecule were further retrieved from Pre-ADMET web-based application [18]. Furthermore, structural analysis of the final lead molecule was also performed with the help of the discovery studio visualizer and PyMOL software.

\section{RESULTS}

\subsection{Active Site of Chloramphenicol Acetyltransferase}

Discovery Studio was used to identify, visualize, and analyze active inhibition sites for chloramphenicol acetyltransferase. For visualizing and identifying the structure of the target, PDB ID 6PXA was retrieved. A two-dimensional structure was then generated containing the receptor and its bound inhibitor. This was then used to identify the bound amino acid residues in the active site of the receptor. Identification of the bound residues was helpful in designing the ligands in such a manner so that they could easily fit into the active pocket of the target. The active site residues of 6PXA PDB identified were Phe 105, Phe 98, Ser 101, Glu 104, Phe 100, Pro 99, Gln 88, Ala 85, Leu 48, Phe 62, Phe 113 , Gly 89 . The gridbox for protein-ligand was set to encase all these active site residues.

\subsection{Florfenicol Docking}

A protein-ligand docking value was obtained between florfenicol and the chloramphenicol acetyltransferase receptor using PyRx (Autodock Vina). This docking score was then used as a base score for further design of the lead molecules. The binding energy obtained for florfenicol was $-6.0 \mathrm{kcal} / \mathrm{mol}$.

\subsection{Primary Ligand Modifications of Florfenicol}

Chemsketch was used to design the primary lead modifications. A total of 15 modifications was developed by altering the $\mathrm{R}$ groups present in the basic framework. These 15 modifications are displayed in Figure 1 and the modifications are summarized in Table 1. Primary modifications were performed to cover the active site pocket of the receptor effectively compared to the basic framework of the lead molecule. The active site of the protein is highlighted in a light green color, as compared to the allosteric portions of the protein.

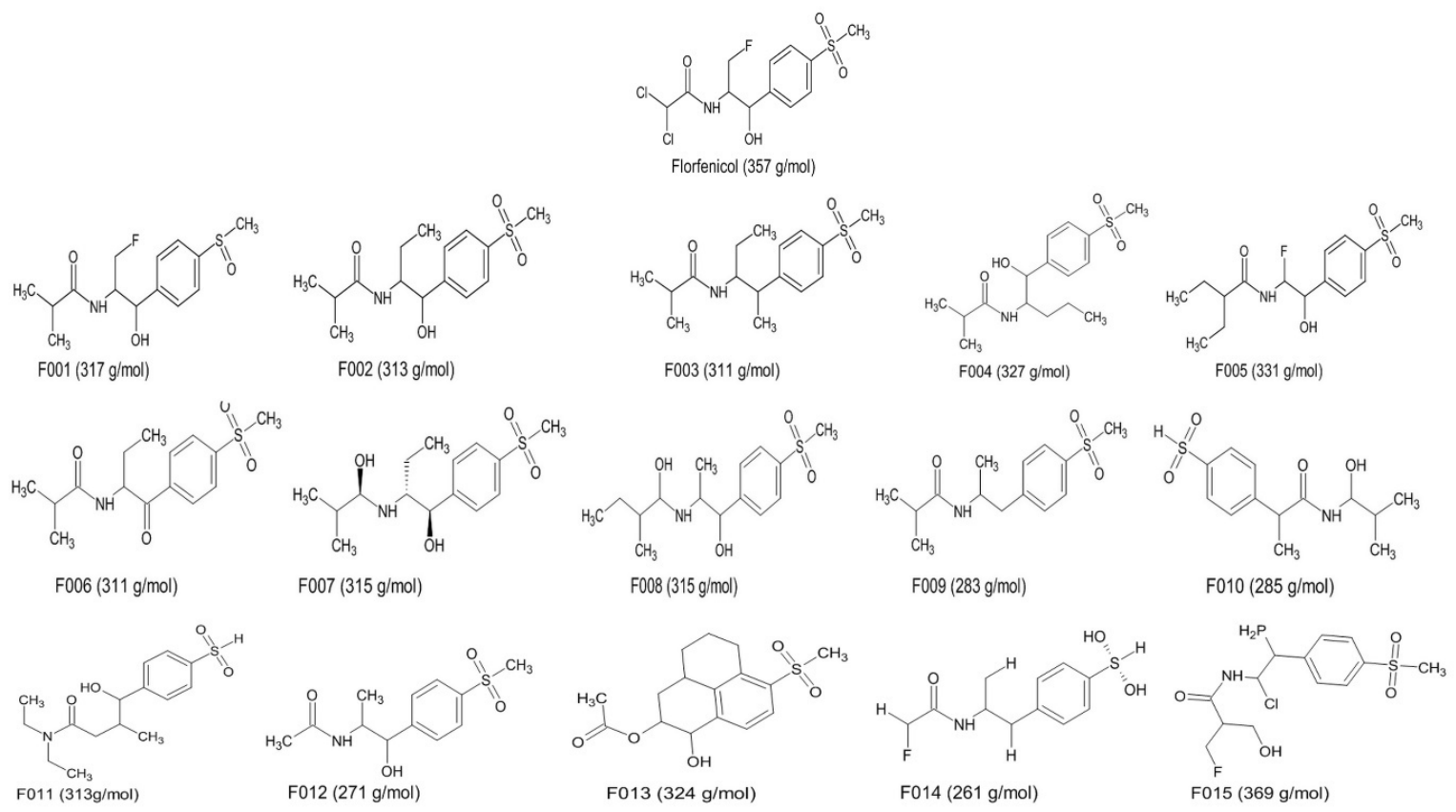

Figure 1: Initial ligand modifications were done using Chemsketch, 15 structures were developed based on modifications. 
Table 1: List of all initially modified ligands.

\begin{tabular}{llc} 
Primary ligand name & \multicolumn{1}{c}{ Changes In florfenicol structure } & Molecular formula \\
F001 & All Cl atoms are replaced by methyl group & $\mathrm{C}_{14} \mathrm{H}_{20} \mathrm{FNO}_{4} \mathrm{~S}$ \\
F002 & Both $\mathrm{Cl}$ and $\mathrm{F}$ are replaced by methyl group & $\mathrm{C}_{15} \mathrm{H}_{23} \mathrm{NO}_{4} \mathrm{~S}$ \\
F003 & All Cl, F, and $\mathrm{OH}$ are replaced by methyl group & $\mathrm{C}_{16} \mathrm{H}_{25} \mathrm{NO}_{3} \mathrm{~S}$ \\
F004 & $\mathrm{Cl}$ is replaced by methyl group, then F is replaced by propyl group. & $\mathrm{C}_{16} \mathrm{H}_{25} \mathrm{NO}_{4} \mathrm{~S}$ \\
F005 & All Cl atoms are replaced by ethyl group & $\mathrm{C}_{15} \mathrm{H}_{22} \mathrm{FNO}_{4} \mathrm{~S}$ \\
F006 & All Cl atoms are replaced by methyl group; F is replaced by ethyl group. Alcohol group is & $\mathrm{C}_{15} \mathrm{H}_{21} \mathrm{NO}_{4} \mathrm{~S}$ \\
F007 & oxidized to keto group. & $\mathrm{C}_{15} \mathrm{H}_{25} \mathrm{NO}_{4} \mathrm{~S}$ \\
F008 & All Cl atoms are replaced by methyl group, F is replaced by Ethyl group & $\mathrm{C}_{15} \mathrm{H}_{25} \mathrm{NO}_{4} \mathrm{~S}$ \\
F009 & One Cl and one F are replaced by methyl group. One Cl is replaced by ethyl group. & $\mathrm{C}_{15} \mathrm{H}_{25} \mathrm{NO}_{4} \mathrm{~S}$ \\
F010 & Both Cl and F are replaced by methyl group. OH, Group is removed. & $\mathrm{C}_{13} \mathrm{H}_{19} \mathrm{NO}_{4} \mathrm{~S}$ \\
F011 & All Cl atoms and OH are replaced by methyl group. Keto group is reduced to alcohol. & $\mathrm{C}_{13} \mathrm{H}_{19} \mathrm{NO}_{4} \mathrm{~S}$ \\
F012 & All Cl atoms are replaced by ethyl group. F is replaced by OH. & $\mathrm{C}_{12} \mathrm{H}_{17} \mathrm{NO}_{4} \mathrm{~S}$ \\
F013 & Dichloromethane group is removed. & $\mathrm{C}_{16} \mathrm{H}_{20} \mathrm{O}_{5} \mathrm{~S}$ \\
F014 & Hexahydrophenalene system is introduced. & $\mathrm{C}_{11} \mathrm{H}_{16} \mathrm{FNO}_{3} \mathrm{~S}$ \\
F015 & One Cl is replaced by F And one F is replaced by H. & Hydroxyl group is replaced by phosphate group. Two Cl is replaced by floro methyl and \\
\hline
\end{tabular}

Table 2: Lipinski's rule of five analysis of florfenicol and primary modified ligands for ADMET properties.

\begin{tabular}{|c|c|c|c|c|c|c|}
\hline Ligand & $C \log P$ & Solubility & MW & TPSA & Drug likeness & Drug score \\
\hline Florfenicol & 0.2 & -2.91 & 357 & 91.85 & 2.55 & 0.11 \\
\hline F001 & 0.24 & -2.64 & 317 & 91.85 & 2.34 & 0.87 \\
\hline F002 & 0.92 & -2.83 & 313 & 91.85 & 4.79 & 0.89 \\
\hline F003 & 2.42 & -3.75 & 311 & 71.62 & 4.53 & 0.81 \\
\hline F004 & 1.38 & -3.1 & 327 & 91.85 & 2.33 & 0.83 \\
\hline F005 & -1 & -3.12 & 331 & 91.85 & 3.67 & 0.86 \\
\hline F006 & 1.1 & -3.56 & 311 & 88.69 & 5.48 & 0.85 \\
\hline F007 & 0.56 & -2.67 & 315 & 95.01 & 3.28 & 0.89 \\
\hline F008 & 0.56 & -2.66 & 315 & 95.01 & 5.89 & 0.9 \\
\hline F009 & 1.54 & -3.11 & 283 & 71.62 & 5.37 & 0.89 \\
\hline F010 & 0.85 & -2.53 & 285 & 83.47 & 2.76 & 0.89 \\
\hline F011 & 1.18 & -2 & 313 & 74.68 & 4.51 & 0.91 \\
\hline F012 & -0.2 & -2.13 & 271 & 91.85 & 3.65 & 0.93 \\
\hline F013 & 1.84 & -3.55 & 324 & 89.05 & -1.32 & 0.51 \\
\hline F014 & -0.9 & -1.98 & 261 & 69.56 & 3.35 & 0.93 \\
\hline F015 & 0.4 & -3.04 & 369 & 91.85 & 0.72 & 0.71 \\
\hline
\end{tabular}

Green: No risk; Yellow: Low risk; Orange: Medium risk; Red: High risk.

\subsection{Primary Lead Development and Drug Screening}

The OSIRIS property explorer tool was used to modify the lead molecule florfenicol. The structure of florfenicol was assessed in the stand-alone Java Runtime Environment. The 15 modified ligands were then designed by substitution or deletion or by addition of atoms in a manner such that the skeleton structure of the lead molecule was retained. The 15 modifications were done basically to increase both solubility and polarity as well as reducing unnecessary side effects and toxicity (Table 2). Using Lipinski's rule of five, ADMET analysis of the 15 modifications was done using physiological parameters such as LogP, solubility, TPSA, molecular weight, drug-likeness, and drug score. All the values were noted. The color code indicated the severity of the unnecessary side effects. The green color indicated no unnecessary side effects. The red color indicated severe side effects. Orange and yellow color denoted medium and low risk of unnecessary side effects respectively. The better property showing designed molecules was then draw in Chemsketch. Following this, the molecules were saved in the PDB format for further docking analysis. All 15 initially modified structures showed better ADME 


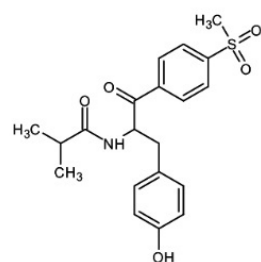

F016 $(389 \mathrm{~g} / \mathrm{mol})$

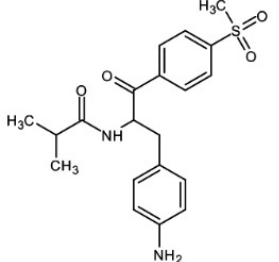

F017 (388 g/mol)

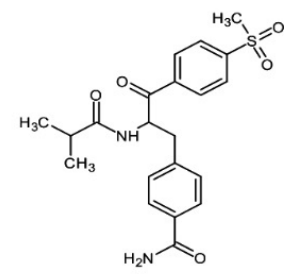

$\mathrm{F} 018(416 \mathrm{~g} / \mathrm{mol})$

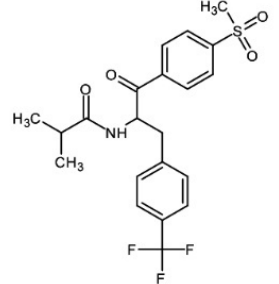

$\mathrm{F019}(441 \mathrm{~g} / \mathrm{mol})$<smiles>CC(C)C(=O)N[C@@H](Cc1ccc(C(=O)O[14CH3])cc1)C(=O)c1ccc(S(C)(=O)=O)cc1</smiles>
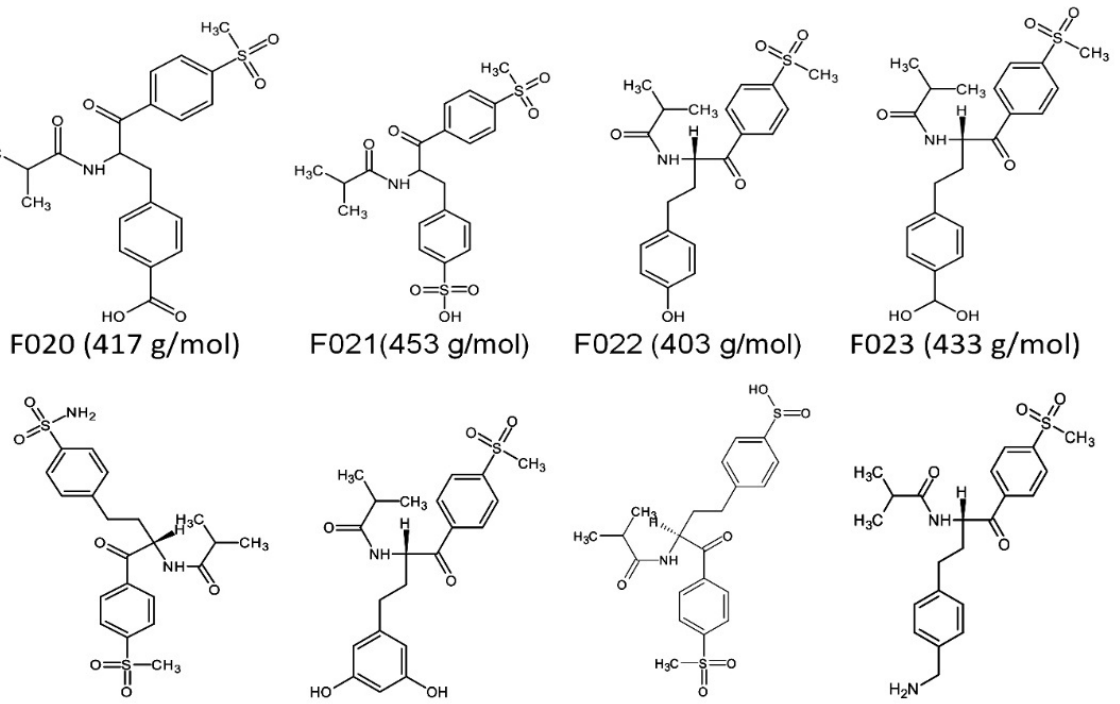

F024 (466 g/mol)

F025 (419 g/mol)

F026 (451 g/mol)

F027 (416 g/mol)

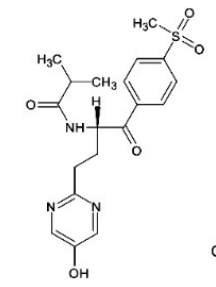

F028 (405 g/mol) F029 (409 g/mol)<smiles>CC(C)C(=O)NC(C(=O)c1ccc(S(C)(=O)=O)cc1)c1ccc(S(C)(=O)=O)cc1</smiles>

F030 $(437 \mathrm{~g} / \mathrm{mol})$<smiles>CC(C)C(=O)N[C@H](C(=O)c1ccc(C(C)(C)C)cc1)c1cc(O)cc(O)c1</smiles>

F031 $(391 \mathrm{~g} / \mathrm{mol})$

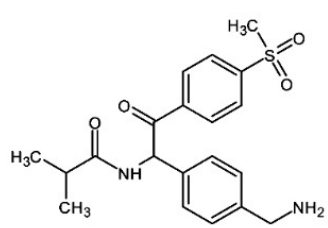

F032 $(388 \mathrm{~g} / \mathrm{mol})$

Figure 2: F006 was taken as the skeletal structure to design 17 secondary structures.

properties than the basic framework florfenicol. Moreover, all the primary modifications, when assessed on OSIRIS property explorer showed zero risks related to mutagenic, tumorigenic, irritant, and reproductive effect factors.

\subsection{Docking Results and Selection of Lead Molecule}

The 15 modifications were now docked with the target chloramphenicol acetyltransferase (Table 3). The docking was performed on PyRx. Out of the 15 modifications, F002, F003, F004, F005, F006, F009, F010, F011, F012, F013, F014, and F015 showed docking scores of $-6.5,-6.5,-6.8,-7.1,-7.3,-6.4$, $-6.7,-6.6,-6.8,-6.9,-6.2$ and $-6.3 \mathrm{kcal} / \mathrm{mol}$ respectively. Out of the 15 initially modified ligands, 12 of the ligands showed higher binding energy than the basic framework. From these 12 ligands, the further selection was made on the basis of higher binding affinity along with better ADME properties. Among these 12 selected ligands, F006 was chosen was for further secondary ligand development as it had the best docking score as well as good ADME properties. Furthermore, F006, when assessed on the Pre-ADMET web server, showed poor blood-brain barrier property with a value of 0.01222 . It also displayed good water solubility property with a value of 19.318 as well as good plasma protein binding property with a value of 77.7702 . Moreover, it also displayed poor skin permeability property with a value of -1.2802 .

\subsection{Secondary Ligand Modifications Using F006}

Using F006 as the framework, secondary modifications were done. The modifications were done using Chemsketch. Better structures were developed by altering R-groups and by stabilizing 
Table 3: Drug screening and docking results for florfenicol and its primary modified ligands.

\begin{tabular}{cccc} 
Ligand & Lipinski's rule of five & Veber rule & $\begin{array}{c}\text { Docked value } \\
\text { (kcal/mol) }\end{array}$ \\
\hline Florfenicol & Passed & Passed & -6 \\
F001 & Passed & Passed & -5.9 \\
F002 & Passed & Passed & -6.5 \\
F003 & Passed & Passed & -6.5 \\
F004 & Passed & Passed & -6.8 \\
F005 & Passed & Passed & -7.1 \\
F006 & Passed & Passed & -7.3 \\
F007 & Passed & Passed & -5.7 \\
F008 & Passed & Passed & -5.7 \\
F009 & Passed & Passed & -6.4 \\
F010 & Passed & Passed & -6.7 \\
F011 & Passed & Passed & -6.6 \\
F012 & Passed & Passed & -6.8 \\
F013 & Passed & Passed & -6.9 \\
F014 & Passed & Passed & -6.2 \\
F015 & Passed & Passed & -6.3 \\
\hline
\end{tabular}

Table 4: List of all secondary modified ligands.

\begin{tabular}{|c|c|c|}
\hline $\begin{array}{c}\text { Secondary } \\
\text { ligand } \\
\text { name }\end{array}$ & Change in the F006 structure & $\begin{array}{l}\text { Molecular } \\
\text { formula }\end{array}$ \\
\hline F016 & Methyl group is replaced by Para phenol & $\mathrm{C}_{20} \mathrm{H}_{23} \mathrm{NO}_{5} \mathrm{~S}$ \\
\hline F017 & Methyl group is replaced by Para aniline & $\mathrm{C}_{20} \mathrm{H}_{24} \mathrm{~N}_{2} \mathrm{O}_{4} \mathrm{~S}$ \\
\hline F018 & Methyl group is replaced by Para benzamide & $\mathrm{C}_{21} \mathrm{H}_{24} \mathrm{~N}_{2} \mathrm{O}_{5} \mathrm{~S}$ \\
\hline F019 & Methyl group is replaced by Para (trifluoromethyl) benzene & $\mathrm{C}_{21} \mathrm{H}_{22} \mathrm{~F}_{3} \mathrm{NO}_{4} \mathrm{~S}$ \\
\hline F20 & Methyl group is replaced by Para Benzoic acid. & $\mathrm{C}_{21} \mathrm{H}_{23} \mathrm{NO}_{6} \mathrm{~S}$ \\
\hline $\mathrm{F} 21$ & Methyl group is replaced by Para benzenesulfonic acid & $\mathrm{C}_{20} \mathrm{H}_{23} \mathrm{NO}_{7} \mathrm{~S}_{2}$ \\
\hline $\mathrm{F} 22$ & Methyl group is replaced by Para hydoxy toluene & $\mathrm{C}_{21} \mathrm{H}_{25} \mathrm{NO}_{5} \mathrm{~S}$ \\
\hline F23 & Methyl group is replaced by Para tolylmethanediol. & $\mathrm{C}_{22} \mathrm{H}_{27} \mathrm{NO}_{6} \mathrm{~S}$ \\
\hline F24 & Methyl group is replaced by 4-(methyl sulfonyl)benzaldehyde & $\mathrm{C}_{21} \mathrm{H}_{26} \mathrm{~N}_{2} \mathrm{O}_{6} \mathrm{~S}_{2}$ \\
\hline F25 & Methyl group is replaced by 5 -ethylbenzene-1,3-diol & $\mathrm{C}_{21} \mathrm{H}_{25} \mathrm{NO}_{6} \mathrm{~S}$ \\
\hline F26 & $\begin{array}{l}\mathrm{SO} 2 \mathrm{CH} 3 \text { group is replaced by } \mathrm{SOOH} \text {, methyl group is replace } \\
\text { by } 4 \text { (methyl sulfonyl) benzaldehyde }\end{array}$ & $\mathrm{C}_{21} \mathrm{H}_{25} \mathrm{NO}_{6} \mathrm{~S}_{2}$ \\
\hline F27 & Methyl group is replaced by (4-ethyl phenyl) methanamine & $\mathrm{C}_{22} \mathrm{H}_{28} \mathrm{~N}_{2} \mathrm{O}_{4} \mathrm{~S}$ \\
\hline F28 & Methyl group is replaced by 2-ethylpyrimidin-5-ol & $\mathrm{C}_{19} \mathrm{H}_{27} \mathrm{~N}_{3} \mathrm{O}_{5} \mathrm{~S}$ \\
\hline F29 & $\begin{array}{l}\text { Methyl group is replaced by -5-ethyl-1,2,3,4- } \\
\text { tetrahydropyrimidin-2-ol }\end{array}$ & $\mathrm{C}_{19} \mathrm{H}_{27} \mathrm{~N}_{3} \mathrm{O}_{5} \mathrm{~S}$ \\
\hline $\mathrm{F} 30$ & Methyl group is replaced by (methyl sulfonyl) benzene & $\mathrm{C}_{20} \mathrm{H}_{23} \mathrm{NO}_{6} \mathrm{~S}_{2}$ \\
\hline $\mathrm{F} 31$ & Methyl group is replaced by resorcinol & $\mathrm{C}_{19} \mathrm{H}_{21} \mathrm{NO}_{6} \mathrm{~S}$ \\
\hline F32 & Methyl group is replaced by Para phenylmethanamine & $\mathrm{C}_{20} \mathrm{H}_{24} \mathrm{~N}_{2} \mathrm{O}_{4} \mathrm{~S}$ \\
\hline
\end{tabular}

hydrophilic hydrophobic interactions. 17 secondary modifications were developed from F006 and are displayed in Figure 2 and the chances are listed in Table 4.

\subsection{Secondary Lead Development and Drug Screening}

The OSIRIS property explorer tool was used to modify the lead molecule F006. The structure of florfenicol was assessed in 


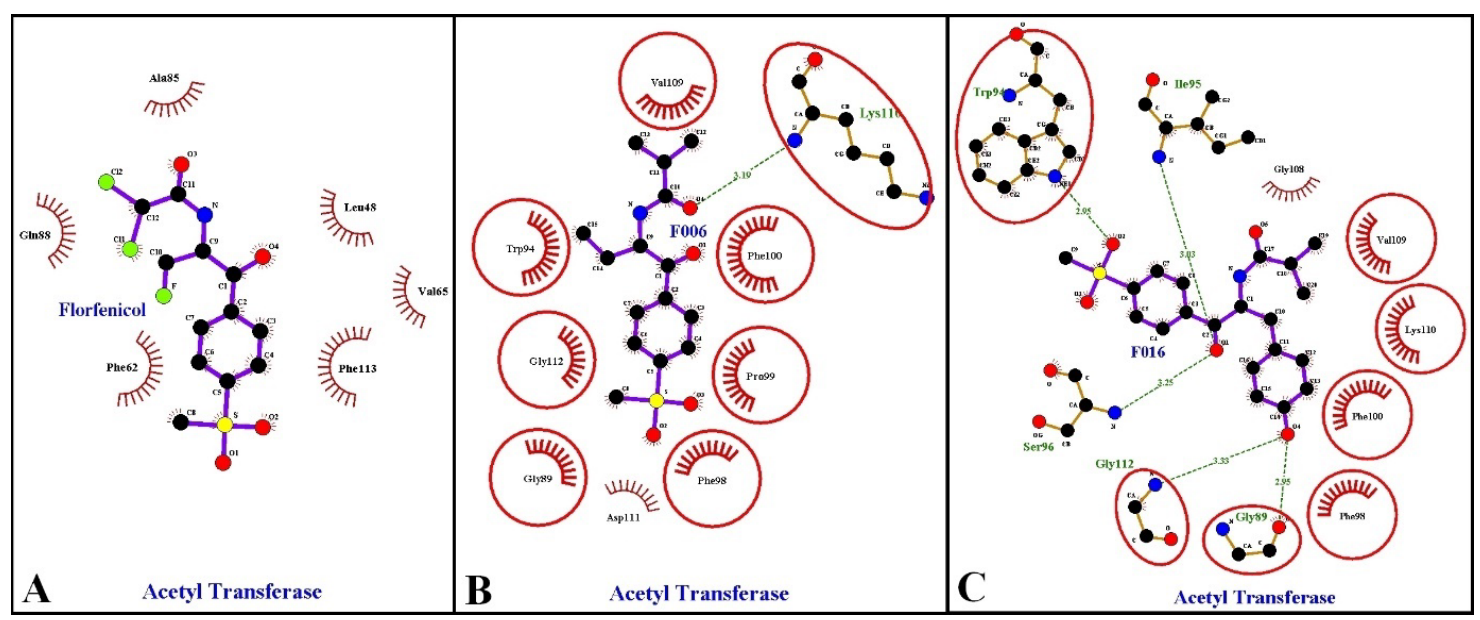

Figure 3: This figure compares 2D amino acid interactions of F016, F06 and florfenicol with the receptor bacterial chloramphenicol acetyltransferase generated with the help of Ligplus application. A represents amino acid interactions of florfenicol, B represents amino acid interactions of F06, C represents amino acid interactions of F016.

Table 5: Lipinski's rule of five analysis of secondary modified ligands for ADMET properties.

\begin{tabular}{ccccccc} 
Ligands & ClogP & Solubility & Mol. Wt & TPSA & Drug likeness & Drug score \\
F016 & 1.74 & -4.13 & 389 & 108.9 & 6.36 & 0.75 \\
F017 & 1.41 & -4.5 & 388 & 114.7 & 5.38 & 0.72 \\
F018 & 1.18 & -4.52 & 416 & 131.7 & 3.91 & 0.69 \\
F019 & 2.94 & -5.2 & 441 & 88.69 & -2.08 & 0.32 \\
F020 & 1.58 & -4.44 & 417 & 125.9 & 0.39 & 0.56 \\
F021 & -0.14 & -3.03 & 453 & 151.4 & 4.16 & 0.46 \\
F022 & 2.2 & -4.4 & 403 & 108.9 & 4.55 & 0.7 \\
F023 & 1.84 & -4.26 & 433 & 129.1 & 2.93 & 0.68 \\
F024 & 1.31 & -4.6 & 466 & 157.2 & 5.24 & 0.63 \\
F025 & 1.85 & -4.1 & 419 & 129.1 & 1.85 & 0.67 \\
F026 & 0.5 & -4.53 & 451 & 145.2 & 4.24 & 0.65 \\
F027 & 1.55 & -4.66 & 416 & 114.7 & 2.77 & 0.66 \\
F028 & 0.53 & -2.68 & 405 & 134.7 & 4.32 & 0.83 \\
F029 & 0.99 & -3.21 & 409 & 132.9 & 5.22 & 0.8 \\
F030 & 0.59 & -4.42 & 437 & 131.2 & 4.48 & 0.68 \\
F031 & 0.9 & -3.42 & 391 & 129.1 & 2.31 & 0.77 \\
F032 & 0.59 & -3.97 & 388 & 114.7 & 4.39 & 0.77 \\
\hline
\end{tabular}

Green: No risk; Yellow: Low risk; Orange: Medium risk; Red: High risk.

the stand-alone Java Runtime Environment. The 17 secondary modified ligands were then designed by substitution or by the addition of atoms in a manner such that the skeleton structure of the lead molecule was retained (Table 5). The 17 modifications were done basically to increase solubility, polarity, binding affinity as well as reducing unnecessary side effects. Using Lipinski's rule of five, ADMET analysis of the 17 secondary modifications was done using physiological parameters such as LogP, solubility, TPSA, molecular weight, drug-likeness, and drug score. All the values were noted. The color code indicated the severity of the unnecessary side effect. The green color indicated no unnecessary side effects. The red color indicated severe side effects. Orange and yellow colors denoted medium and low risk of unnecessary side effects respectively. The better properties showing designed molecules were then drawn in Chemsketch. Following this, the molecules were saved in PDB format for further docking analysis. All secondary modifications, when assessed on OSIRIS property explorer showed zero risks related to mutagenic, tumorigenic, irritant, and reproductive effect factors except F021, which showed a high risk of mutagenicity.

\subsection{Docking Results and Selection of Lead Molecule}

The 17 new secondary modified ligands were now docked with chloramphenicol acetyltransferase (Table 6). All the secondary modified ligands showed better docking scores than the basic 
Table 6: Drug screening and docking results of secondary modified ligands.

\begin{tabular}{cccc} 
Ligand & Lipinski's rule of five & Veber rule & Docked value \\
F016 & Passed & Passed & -8.6 \\
F017 & Passed & Passed & -8.5 \\
F018 & Passed & Passed & -9.3 \\
F019 & Passed & Passed & -9.2 \\
F020 & Passed & Passed & -8.3 \\
F021 & Passed & Failed & -8.8 \\
F022 & Passed & Passed & -8.4 \\
F023 & Passed & Passed & -8 \\
F024 & Passed & Failed & -8.7 \\
F025 & Passed & Passed & -8.4 \\
F026 & Passed & Failed & -8.5 \\
F027 & Passed & Passed & -8.4 \\
F028 & Passed & Passed & -8.1 \\
F029 & Passed & Passed & -8.1 \\
F030 & Passed & Passed & -8.5 \\
F031 & Passed & Passed & -8.6 \\
F032 & Passed & Passed & -8.4 \\
\hline
\end{tabular}

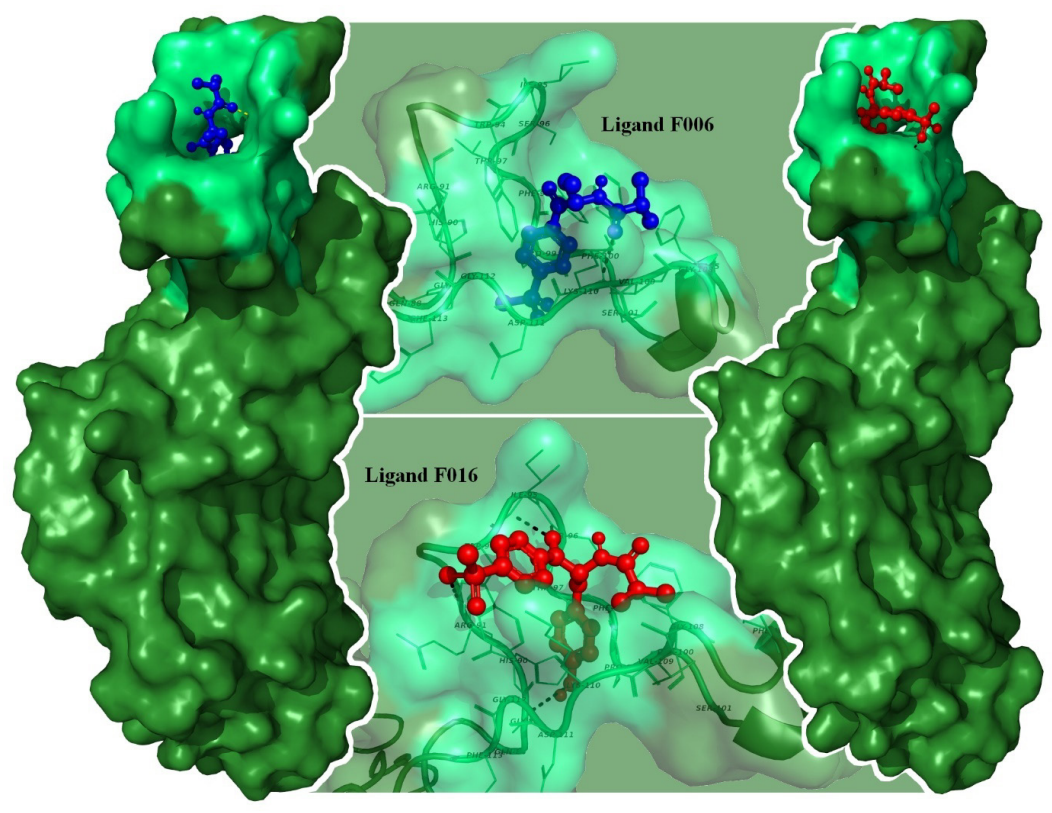

Figure 4: PyMOL visualization of chloramphenicol acetyltransferase with F016 (red) and F06 (blue). The apoprotein is represented by dark green color. The active sites are represented by light green color.

framework F006. But among these F016 was chosen as it showed a better docking score of $-8.6 \mathrm{kcal} / \mathrm{mol}$ along with good ADME properties. More drug properties of F016 were further retrieved from Pre-ADMET web-based application. F016, when assessed on the Pre-ADMET web server showed poor blood-brain Barrier property with a value of 0.02242 . It also has good plasma protein binding property with a value of 86.2747 , good water solubility property with a value of 10.4255 , and poor skin permeability property with a value of -1.6579 . Structural analysis of F016 was also performed using ligplus and PyMOL software.

\section{DISCUSSION}

Vibriosis has been considered a major problem worldwide in the aquaculture industry. Moreover, there has not been much qualitative research on vibriosis or organisms responsible for causing vibriosis. With the help of this study, we were able to 
design a molecule for targeting chloramphenicol acetyltransferase, a major enzyme present in vibriosis-causing bacteria, which is responsible for providing resistance against antibiotics.

With the help of the literature survey, we have found out studies that have clearly stated bacterial chloramphenicol acetyltransferase's importance in vibrio species. The literature survey has also helped us to isolate our main basic framework, florfenicol. The basic framework of florfenicol was modified to produce 15 primary structures and 17 secondary structures. These modified structures were then subjected to docking, screening, and ADME analysis. We were able to design a molecule, F016 with the help of this study which has shown good binding affinity with bacterial chloramphenicol acetyltransferase receptor along with good ADME properties (Figure 3 and Figure 4). The F016 also showed similar interactions with bacterial acetyltransferase like florfenicol, thereby making it an ideal molecule to inhibit chloramphenicol acetyltransferase. Lastly, we can conclude by saying that F016 can be considered as a lead candidate in binding with chloramphenicol acetyltransferase, and hence can be used for treating vibriosis.

\section{CONCLUSION}

The results of this study conclude that the drug target chloramphenicol acetyltransferase protein can be used for developing antibiotics against vibriosis infection in aquaculture management. Florfenicol structure was taken as a skeleton and was modified to develop 15 ligands that were screened against the drug target. F006 was used as skeleton structure for secondary modification. Among all the developed modifications of the ligand, the ligand molecule F016 ligand was identified as the best modified ligand that shows greater inhibition potential against chloramphenicol acetyltransferase protein and demonstrates significantly better ADME property. This molecule should further be investigated in-vitro to justify the given hypothesis.

\section{ACKNOWLEDGMENTS}

The authors thank SciWris Life Sciences for supporting this study.

\section{CONFLICT OF INTERSET}

No known conflict of interest for this study.

\section{FUNDING}

No funding was availed for this study.

\section{ETHICAL APPROVALS}

This study does not involve experiments on animals or human subjects.

\section{REFERENCES}

1. Mohamad N, Amal MNA, Yasin ISM, Saad MZ, Nasruddin NS, Alsaari $\mathrm{N}$, et al. Vibriosis in cultured marine fishes: a review. Aquaculture 2019;512(May):1-17.

2. Dunn AK. Vibrio fischeri metabolism: symbiosis and beyond [Internet]. 1st edition. In: Advances in Microbial Physiology, Elsevier Ltd, Amsterdam, Netherlands, pp 37-68, vol 61, 2012.
3. Seljestokken B, Bergh, Melingen GO, Rudra H, Hetlelid Olsen R, Samuelsen OB. Treating experimentally induced vibriosis (Listonella anguillarum) in cod, Gadus morhua L., with florfenicol. J Fish Dis 2006;29(12):737-42.

4. Samuelsen OB, Bergh Ø. Efficacy of orally administered florfenicol and oxolinic acid for the treatment of vibriosis in cod (Gadus morhua). Aquaculture 2004;235(1-4):27-35.

5. Wang L, Han YN, Jin S, Ma Y, Wang GL, Zhao QS, et al. Pharmacokinetic study of florfenicol in healthy and vibriosis-infected pseudosciaena crocea after oral administration. J Appl Biol Chem 2015;58(4):363-8.

6. Parmar P, Yusufzai SKI, Parmar H V, Nanjiyani RP, Chavda VM. Therapeutic potentiality of florfenicol against vibriosis in Litopenaeus vannamei therapeutic potentiality of florfenicol against vibriosis in Litopenaeus vannamei. J Entomol Zool Stud 2018;6(August):463-7.

7. Jangaran Nejad A, Peyghan R, Najafzadeh Varzi H, Shahriyari A. Florfenicol pharmacokinetics following intravenous and oral administrations and its elimination after oral and bath administrations in common carp (Cyprinus carpio). Vet Res forum an Int Q J 2017;8(4):327-31.

8. Convention USP. FLORFENICOL (Veterinary-Systemic) pharmacology/pharmacokinetics. United States Pharmacopeial Convention, North Bethesda, MD, pp 1-6, 2007.

9. Papich MG DVM, MS, DACVCP. Florfenicol. In: Saunders Handbook Veterinary Drugs, St.Louis, MO: Saunders Elsevier, pp 327-9, 2016.

10. Martos P, Shurmer B. Sample preparation techniques for the determination of veterinary drugs in food matrices [Internet] In: Comprehensive Sampling and Sample Preparation, Elsevier, Amsterdam, Netherlands, pp 405-14, vol 4, 2012.

11. Sreenivas A, Menta V, Ravi L. Designing of ligand-107 an effective variant of antimalarial drug lumefantrine through structure-based computer-aided drug development approach. Asian. J Pharm 2020;14(36):2-9.

12. Sadeesh N, Camil Rex M, Ravi L. Structure-based drug designing for n-methyl-d-aspartate receptor: link between neurodegenerative disease and glioblastoma. J Appl Biol Biotechnol 2021;9(1):25-31.

13. DeLano WL. The PyMOL molecular graphics system, version 2.3 . Schrödinger LLC, New York, NY, 2020.

14. Seeliger D, De Groot BL. Ligand docking and binding site analysis with PyMOL and Autodock/Vina. J Comput Aided Mol Des 2010;24(5):417-22.

15. Visualizer DS. v4. 0.100. 13345. Accelrys Software Inc, San Diego, CA, 2005.

16. Studio D. Dassault systemes BIOVIA, siscovery studio modelling environment, release 4.5. Accelrys Software Inc, San Diego, CA, 2015

17. Dallakyan S, Olson AJ. Small-molecule library screening by docking with PyRx. Methods Mol Biol 2015;1263:243-50.

18. Lee SK, Lee IH, Kim HJ, Chang GS, Chung JE, No KT. The PreADME approach: web-based program for rapid prediction of physicochemical, drug absorption and drug-like properties. EuroQSAR 2002 Des Drugs Crop Prot Process Probl Solut [Internet] 2002;418-20. Available via https://preadmet.bmdrc.kr/toxicity-prediction/

How to cite this article: Bhowmik R, Roy S, Sengupta S, Ravi L. Computer aided drug design of florfenicol to target chloramphenicol acetyltransferase of vibriosis causing pathogens. J Appl Biol Biotech 2022; 10(01):76-84. 